\title{
Self-Similar Collapse of Scalar Field with Plane Symmetry
}

\author{
Anzhong Wang ${ }^{a, b *}$, Yumei $\mathrm{Wu}^{a, c \dagger}{ }^{\dagger}$, Zhong Chao $\mathrm{Wu}^{a \ddagger}$ \\ ${ }^{a}$ Department of Physics, Zhejiang University of Technology, Hang Zhou 310032, P.R. China \\ ${ }^{b}$ CASPER, Physics Department, P.O. Box 97316, Baylor University, Waco, TX76798-7316 \\ ${ }^{c}$ Institute of Mathematics, the Federal University of Rio de Janeiro, Caixa Postal 68530, CEP 21945-970, Rio de Janeiro, \\ RJ, Brazil
}

(October 2, 2018)

\begin{abstract}
Plane symmetric self-similar solutions to Einstein's four-dimensional theory of gravity are studied and all such solutions are given analytically in closed form. The local and global properties of these solutions are investigated and it is shown that some of the solutions can be interpreted as representing gravitational collapse of the scalar field. During the collapse, trapped surfaces are never developed. As a result, no black hole is formed. Although the collapse always ends with spacetime singularities, it is found that these singularities are spacelike and not naked.
\end{abstract}

PACS Numbers: 04.20.Dw 04.20.Jb 04.40.Nr 97.60.Lf

\section{INTRODUCTION}

Recently, self-similar solutions to the Einstein field equations have attracted lots of attention, not only because the corresponding problem can be considerably simplified and, as a result, can be studied analytically [1], but also because their relevance to critical phenomena in gravitational collapse, which were first discovered by Choptuik in 1993 in his numerical study of collapsing spherically symmetric scalar field [2]. The phenomena have lately been found in various matter fields collapse [3,4]. In particular, one of the present authors studied analytically the gravitational collapse of cylindrically symmetric scalar field in four-dimensional spacetimes, and found a class of exact solutions with self-similarity [5]. It was shown explicitly that one of the solutions has precisely one unstable mode. By definition, this is a critical solution that sits on the boundary separating two different phases in the phase space of the initial data. This serves as the second analytical model of critical collapse found so far. The first analytical model was found by Garfinkle in 2001 in $2+1$ gravity [6], right after the numerical simulations of Pretorius and Choptuik [7] and Husain and Olivier [8]. The linear perturbations of Garfinkle's self-similar solutions, denoted by $S[n]$, where $n$ is a parameter, were systematically studied in [9] and [10]. Due to different boundary conditions imposed, different results were obtained. In particular, in [9] it was found that the solution with $n=3$ has precisely one unstable mode, while in [10] it was found that the one with $n=4$ has one and only one unstable mode. Although the solution with $n=4$ is best matched with the numerical critical solution found by Pretorius and Choptuik, the exponent, $\gamma$, of the black hole mass

$$
M_{B H} \propto\left(p-p^{*}\right)^{\gamma},
$$

obtained by [11]

$$
\gamma=\frac{1}{\left|k_{1}\right|}
$$

is quite different from the numerical one, $\gamma \sim 1.2[10]$, where $k_{1}$ is the unstable mode of the critical solution. On the other hand, the critical solution found in [9] is different from the numerical one [7], but the resultant exponent, $\gamma=4 / 3$, is close to the numerical one found by Pretorius and Choptuik, although it is still quite different from the numerical one found by Husain and Olivier, $\gamma \sim 0.81[8]$.

In this paper, we have no tendency to resolve the above disputations, but look for more analytical solutions that might represent critical collapse. In the analytical studies of critical collapse, the investigation is usually divided into

\footnotetext{
${ }^{*}$ E-Mail: Anzhong_Wang@baylor.edu

${ }^{\dagger}$ E-Mail: yumei@dmm.im.ufrj.br

${ }^{\ddagger}$ E-Mail: zcwu@zjut.edu.cn
} 
two steps: One first finds some particular solutions by imposing certain symmetries, such as, homothetic self-similarity (HSS). This can mathematically simplify the problem considerably. For example, in the spherically symmetric case, by imposing HSS symmetry the Einstein field equations will be reduced from PDE's to ODE's. Once the particular solutions are known, one can study their linear perturbations and find the spectra of the corresponding eigen-modes. If a solution has precisely one unstable mode, it may represent a critical solution, sitting on a boundary that separates two different basins of attraction in the phase space. In this paper, we shall restrict ourselves only to the first step. In particular, we shall study self-similar solutions of massless scalar field with plane symmetry in Einstein's fourdimensional theory of gravity, and present all such solutions in closed form. Then, we shall study their local and global properties, whereby show some of them can be interpreted as representing gravitational collapse of the scalar field. During the collapse, trapped surfaces are never developed. As a result, no black hole is formed. Although the collapse always ends with spacetime singularities, it is found that these singularities are spacelike and not naked.

\section{PLANE SYMMETRIC SPACETIMES WITH SELF-SIMILARITY}

The general metric for spacetimes with plane symmetry can be cast in the form [12],

$$
d s^{2}=2 e^{-M(u, v)} d u d v-e^{-U(u, v)}\left(d x^{2}+d y^{2}\right)
$$

where $\left\{x^{\mu}\right\}=\{u, v, x, y\},(\mu=0,1,2,3)$, and $-\infty<x^{\mu}<+\infty$. The three Killing vectors that characterize the symmetry are given by $\xi_{(1)}=\partial_{x}, \xi_{(2)}=\partial_{y}$ and $\xi_{(3)}=x \partial_{y}-y \partial_{x}$. The non-vanishing components of the Ricci tensor are given by [13]

$$
\begin{aligned}
R_{u u} & =\frac{1}{2}\left(2 U_{, u u}-U_{, u}^{2}+2 U_{, u} M_{, u}\right), \\
R_{u v} & =\frac{1}{2}\left(2 M_{, u v}+2 U_{, u v}-U_{, u} U_{, v}\right), \\
R_{v v} & =\frac{1}{2}\left(2 U_{, v v}-U_{, v}^{2}+2 U_{, v} M_{, v}\right), \\
R_{x x} & =R_{y y}=-e^{M-U}\left(U_{, u v}-U_{, u} U_{, v}\right),
\end{aligned}
$$

where ()$_{, u} \equiv \partial() / \partial_{u}$ and so on. A massless scalar field satisfies the Klein-Gordon equation, $g^{\alpha \beta} \phi_{; \alpha \beta}=0$, which in the present case takes the form,

$$
2 \phi_{, u v}-\phi_{, u} U_{, v}-\phi_{, v} U_{, u}=0
$$

However, this equation is not independent of the Einstein field equations and can be obtained from the Bianchi identities $G_{\mu \alpha ; \beta} g^{\alpha \beta}=0$.

Spacetimes with homothetic self-similarity (or self-similarity of the first kind) is usually defined by the existence of a conform Killing vector $\xi^{\mu}$ that satisfies the equations [14],

$$
\xi_{\mu ; \nu}+\xi_{\nu ; \mu}=2 g_{\mu \nu}
$$

where a semicolon ";" denotes the covariant derivative. It can be shown [15] that for the spacetimes described by Eq.(2.1) there are two types of self-similar solutions. The first type is given by

$$
\begin{aligned}
& M(u, v)=M(z), \quad U(u, v)=S(z)-2 \ln (-u), \\
& \xi^{\mu} \partial_{\mu}=u \partial_{u}+v \partial_{v}, \quad z \equiv \frac{v}{(-u)},
\end{aligned}
$$

while the second type is given by

$$
\begin{aligned}
& M(u, v)=M(z), \quad U(u, v)=U(z), \\
& \xi^{\mu} \partial_{\mu}=x^{\lambda} \partial_{\lambda}, \quad z \equiv \frac{v}{(-u)}
\end{aligned}
$$

Solutions with the properties of Eq.(2.8) will be referred to as the Type A self-similar solutions, and the ones with the properties of Eq.(2.9) will be referred to as the Type B self-similar solutions. In the following we shall consider them separately. For the sake of convenience, we shall first restrict ourselves to the region $u \leq 0, v \geq 0$. Once we find the self-similar solutions in this region, we shall extend them to other regions whenever it is necessary. 


\section{SOLUTIONS OF MASSLESS SCALAR FIELD WITH TYPE A SELF-SIMILARITY}

The solutions with Type A self-similarity are characterized by Eq.(2.8), for which the non-vanishing components of the Ricci tensor given by Eqs.(2.2)-(2.5) become

$$
\begin{aligned}
& R_{u u}=\frac{z}{u^{2}}\left[z\left(S^{\prime \prime}-\frac{1}{2} S^{\prime 2}\right)+\left(z S^{\prime}+2\right) M^{\prime}\right], \\
& R_{u v}=\frac{1}{u^{2}}\left[z\left(M^{\prime \prime}+S^{\prime \prime}\right)-\frac{1}{2} z S^{\prime 2}+M^{\prime}\right], \\
& R_{v v}=\frac{1}{u^{2}}\left(S^{\prime \prime}-\frac{1}{2} S^{\prime 2}+S^{\prime} M^{\prime}\right), \\
& R_{x x}=R_{y y}=-\frac{1}{u^{2}} e^{M-U}\left[z\left(S^{\prime \prime}-S^{\prime 2}\right)-S^{\prime}\right],
\end{aligned}
$$

where a prime denotes the ordinary differentiation with respect to $z$. For a massless scalar field, the Einstein field equations read

$$
R_{\mu \nu}=\kappa \phi_{, \mu} \phi_{, \nu}
$$

where $\kappa\left[\equiv 8 \pi G / c^{4}\right]$ is the Einstein coupling constant. In this paper we shall choose units such that $\kappa=1$. It can be shown that a massless scalar field $\phi(u, v)$ that is consistent with spacetimes with homothetic self-similarity must take the form,

$$
\phi(u, v)=2 q \ln (-u)+\varphi(z),
$$

where $q$ is an arbitrary constant, and $\varphi(z)$ is an arbitrary function, which will be determined by the Einstein field equations (3.5). Inserting Eqs.(3.1)-(3.4) and (3.6) into Eq.(3.5), we find that

$$
\begin{aligned}
z\left[z\left(S^{\prime \prime}-\frac{1}{2} S^{2}\right)+\left(z S^{\prime}+2\right) M^{\prime}\right] & =\left(z \varphi^{\prime}-2 q\right)^{2} \\
z\left(M^{\prime \prime}+S^{\prime \prime}\right)-\frac{1}{2} z S^{\prime 2}+M^{\prime} & =\varphi^{\prime}\left(z \varphi^{\prime}-2 q\right), \\
S^{\prime \prime}-\frac{1}{2} S^{2}+S^{\prime} M^{\prime} & =\varphi^{\prime 2} \\
\frac{S^{\prime \prime}}{S^{\prime}}-S^{\prime}-\frac{1}{z} & =0 .
\end{aligned}
$$

Eq.(3.10) has the general solution,

$$
S(z)=-\ln \left|a z^{2}+b\right|
$$

where $a$ and $b$ are two integration constants. When $a b=0$, it can be shown that the corresponding solutions correspond to null dust fluid, and in this paper we shall not consider this case. Thus, from now on we shall assume that $a b \neq 0$. Substituting the solution (3.11) into Eqs.(3.7)-(3.9), we find that

$$
\begin{aligned}
& M^{\prime}=-\frac{1}{2 a z\left(a z^{2}+b\right)}\left[2 a b+\left(a z^{2}+b\right)^{2} \varphi^{\prime 2}\right], \\
& a\left(z \varphi^{\prime}-2 q\right)^{2}+b \varphi^{\prime 2}=-\frac{2 a b}{a z^{2}+b}, \\
& z M^{\prime \prime}+M^{\prime}-\frac{2 a b z}{\left(a z^{2}+b\right)^{2}}=\varphi^{\prime}\left(z \varphi^{\prime}-2 q\right) .
\end{aligned}
$$

On the other hand, one can show that Eq.(2.6) takes the form

$$
\varphi^{\prime \prime}+\frac{2 a z}{a z^{2}+b} \varphi^{\prime}-\frac{2 a q}{a z^{2}+b}=0 .
$$

It can be shown that Eq.(3.14) is not independent. As a matter of fact, it can be obtained from Eqs.(3.12) and (3.15). Thus, in the following we need only to consider Eqs.(3.12), (3.13) and (3.15). Integrating Eq.(3.15) we find that 


$$
\varphi^{\prime}(z)=\frac{2 a q z+c_{1}}{a z^{2}+b}
$$

where $c_{1}$ is a constant. Inserting Eq.(3.16) into Eq.(3.12), we find that the constant $c_{1}$ must be given by

$$
c_{1}= \pm\left[-2 a b\left(1+2 q^{2}\right)\right]^{1 / 2} .
$$

To have $c_{1}$ real, we must require $a b<0$. Then, from Eqs.(3.12) and (3.16) we obtain,

$$
\begin{gathered}
M(z)=2 q^{2} \ln \left|\frac{z}{a z^{2}+b}\right|-c q \ln \left|\frac{\alpha-z}{\alpha+z}\right|+M_{0}, \\
\varphi(z)=q \ln \left|a z^{2}+b\right|+\frac{1}{2} c \ln \left|\frac{\alpha-z}{\alpha+z}\right|+\varphi_{0},
\end{gathered}
$$

where $M_{0}$ and $\varphi_{0}$ are other integration constants, and

$$
\begin{aligned}
c & \equiv \pm\left[2\left(1+2 q^{2}\right)\right]^{1 / 2} \\
\alpha & \equiv\left(-\frac{b}{a}\right)^{1 / 2}, \quad a b<0 .
\end{aligned}
$$

Rescaling the coordinates $x$ and $y$, without lose of generality, we can always set $|a|=1$. Then, the solutions can be finally written in the form,

$$
\begin{aligned}
& M(z)=2 q^{2} \ln \left|\frac{z}{\alpha^{2}-z^{2}}\right|-c q \ln \left|\frac{\alpha-z}{\alpha+z}\right|+M_{0}, \\
& S(z)=-\ln \left|\alpha^{2}-z^{2}\right|, \\
& \varphi(z)=q \ln \left|\alpha^{2}-z^{2}\right|+\frac{1}{2} c \ln \left|\frac{\alpha-z}{\alpha+z}\right|+\varphi_{0} .
\end{aligned}
$$

To study the above solutions, let us first consider the region where $\alpha \geq z$. Then, it can be shown that

$$
\begin{aligned}
\phi_{, \alpha} \phi^{, \alpha}= & \frac{4 \alpha e^{M_{0}}(-u v)^{2 q^{2}}}{[\alpha(-u)-v]^{2 q^{2}+c q+2}[\alpha(-u)+v]^{2 q^{2}-c q+2}} \\
& \times\left[c q\left(v^{2}+\alpha^{2} u^{2}\right)-\alpha\left(1+4 q^{2}\right) u v\right], \\
e^{M}= & \frac{e^{M_{0}}(-u v)^{2 q^{2}}}{[\alpha(-u)-v]^{2 q^{2}+c q}[\alpha(-u)+v]^{2 q^{2}-c q}}, \quad(\alpha \geq z) .
\end{aligned}
$$

Since $2 q^{2} \pm c q+2$ is always positive, from the above expressions we can see that the spacetime is singular on the hypersurface $v=\alpha(-u)$. The normal vector to this surface is given by

$$
n_{\mu} \equiv \frac{\partial(v+\alpha u)}{\partial x^{\mu}}=\delta_{\mu}^{v}+\alpha \delta_{\mu}^{u}
$$

which is always timelike, as now we have $n_{\alpha} n^{\alpha}=2 \alpha e^{M}>0$. Therefore, the singularity is spacelike and services as the up boundary of the region $v \geq 0, u \leq 0$. On the other hand, from these expressions we can also see that the spacetime is free of curvature singularity on the hypersurface $v=0$, although the metric coefficient $M$ is singular. Thus, to have a geodesically maximal spacetime, the solutions need to be extended beyond this surface. For the sake of convenience, in the following we shall consider the two cases $2 q^{2}<1$ and $2 q^{2} \geq 1$ separately.

$$
\text { A. } \alpha \geq z, 2 q^{2}<1
$$

In this case, introducing two new coordinates $\bar{u}$ and $\bar{v}$ via the relations

$$
u=-(-\bar{u})^{n}, \quad v=\bar{v}^{n},
$$


where

$$
n \equiv \frac{1}{1-2 q^{2}},
$$

we find that in terms of $\bar{u}$ and $\bar{v}$ the metric takes the form,

$$
\begin{aligned}
d s^{2}= & {\left[\alpha(-\bar{u})^{n}-\bar{v}^{n}\right]^{2 q^{2}+c q}\left[\alpha(-\bar{u})^{n}+\bar{v}^{n}\right]^{2 q^{2}-c q} d \bar{u} d \bar{v} } \\
& -\left(\alpha^{2} \bar{u}^{2 n}-\bar{v}^{2 n}\right)\left(d x^{2}+d y^{2}\right)
\end{aligned}
$$

from which we can see that the metric coefficients are no longer singular on the hypersurface $v=0$ or $\bar{v}=0$. The coordinate transformations (3.23) map the region $v \geq 0, u \leq 0$ into the region $\bar{v} \geq 0$, $\bar{u} \leq 0$, which will be referred to as Region $I$, as shown in Fig. 1. Then, the region $\bar{v}<0, \bar{u} \leq 0$ represents an extended region, which will be referred to as Region $I I$. It should be noted that this extension is not always physically acceptable. In particular, the extension may not be analytical and the extended metric coefficients may not be real, unless $n$ is an integer. When the extension is not analytical, it is also not unique. Therefore, to have an unique extension, in the following we shall consider the case where $n$ is an integer. Then, in terms of $\bar{v}$ and $\bar{u}$ we find that

$$
\begin{aligned}
\phi_{, \alpha} \phi^{, \alpha}= & \frac{4 \alpha e^{M_{0}}(-\bar{u} \bar{v})^{n-1}}{\left[\alpha(-\bar{u})^{n}-\bar{v}^{n}\right]^{2 q^{2}+c q+2}\left[\alpha(-\bar{u})^{n}+\bar{v}^{n}\right]^{2 q^{2}-c q+2}} \\
& \times\left[c q\left(\bar{v}^{2 n}+\alpha^{2} \bar{u}^{2 n}\right)+\alpha\left(1+4 q^{2}\right)(-\bar{u} \bar{v})^{n}\right],
\end{aligned}
$$

from which we find that $\phi_{, \alpha}$ is timelike only when $n$ is an odd integer in the extended region, $I I$. The spacetime is always singular on the hypersurface $\bar{v}=\alpha^{-1 / n} \bar{u}$. This singularity is timelike, and the corresponding Penrose diagram is given by Fig. 1. Due to this singular behavior, it is found difficult to interpreted the corresponding solutions as representing physically reasonable model of gravitational collapse.

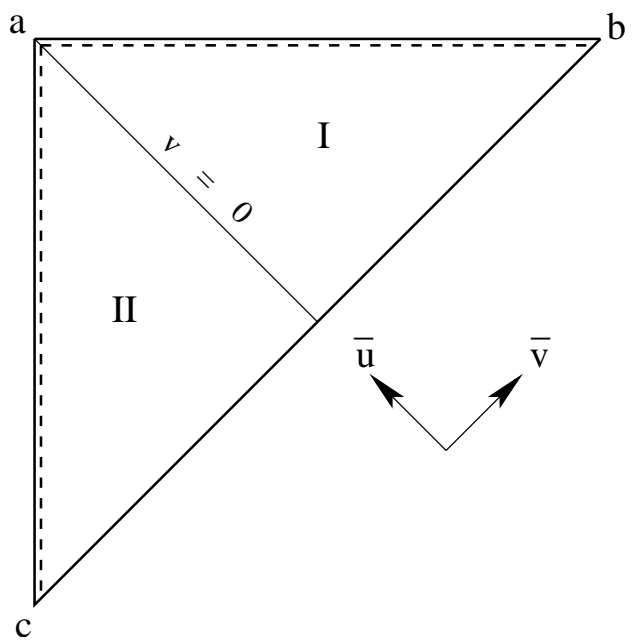

FIG. 1. The Penrose diagram for the solutions given by Eq. (3.25) with $n(>1)$ being an integer. Region $I$ is defined as $v \geq 0, u \leq 0$, and Region $I I$ as $v \leq 0, u \leq 0$. The spacetime is singular on the both horizontal and vertical double lines $a b$ and $a c$, where $v=\alpha(-u)$ and $\bar{v}=\alpha^{-1 / n} \bar{u}$, respectively. The line $b c$ represents the past null infinity $u=-\infty$.

$$
\text { B. } \alpha \geq z, 2 q^{2} \geq 1
$$

In this case, the spacetime in the region $v \geq 0, u \leq 0$ is already geodesically maximal, and does not need to be extended beyond the hypersurface $v=0$. To see this, let us consider the null geodesics along $u, x, y=C o n s t$., which are given by

$$
\frac{d^{2} v}{d \lambda^{2}}-\frac{\partial M\left(u_{0}, v\right)}{\partial v}\left(\frac{d v}{d \lambda}\right)^{2}=0
$$


where $\lambda$ denotes the affine parameter along the null geodesics. Near the hypersurface $v=0$, it can be shown that the above equation has the following general solution,

$$
v(\lambda)= \begin{cases}\left(c_{1} \lambda+c_{2}\right)^{-1 /\left(2 q^{2}-1\right)}, & 2 q^{2}>1 \\ e^{c_{1} \lambda+c_{2}} & 2 q^{2}=1\end{cases}
$$

where $c_{1,2}$ are the integration constants. Thus, as $v \rightarrow 0$, we have $|\lambda| \rightarrow \infty$, that is, the "distance" between the point $(u, v)=\left(u_{0}, 0\right)$ and any other one along the null geodesic $u=u_{0}$, say, $(u, v)=\left(u_{0}, v_{0}\right)$, where $v_{0}>0$, is infinitively large. In other words, the hypersurface $v=0$ in the present case represents a physical boundary of the spacetime. Then, the corresponding Penrose diagram is given by Fig. 2.

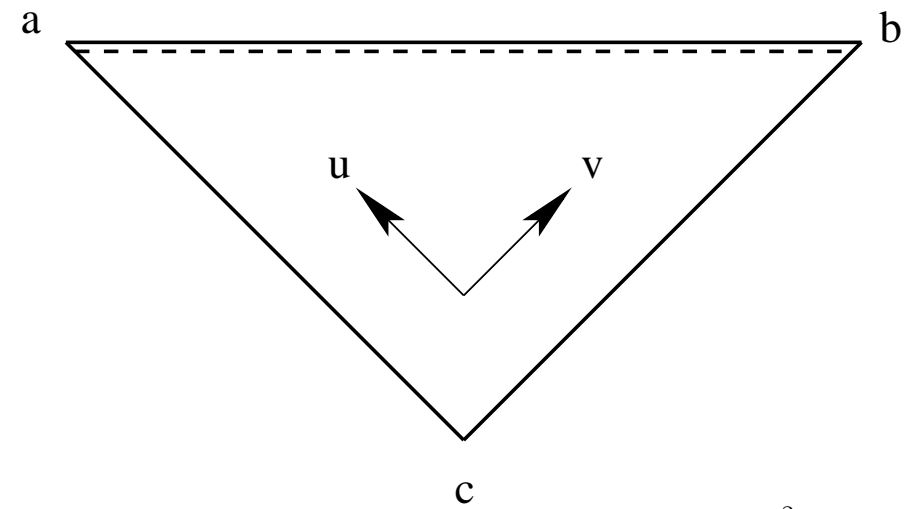

FIG. 2. The Penrose diagram for the solutions given by Eq. (3.20) with $\alpha>z, 2 q^{2}>1$. The spacetime is singular on the horizontal double line $a b$, but not on the ones $a c$ and $b c$ that now serve as the null infinity boundaries of the spacetime, where the line $a c$ is given by $v=0$ and the one $b c$ by $u=-\infty$.

In the region $u<0, v>0$, Eq.(3.21) shows that the scalar field is timelike. Thus, one may consider the above solution as representing gravitational collapse of the scalar field, and the collapse always forms a spacetime singularity on the hypersurface $v=\alpha(-u)$. Although no trapped surface is formed, the singularity is not naked, as it can be seen from Fig. 2.

\section{SOLUTIONS OF MASSLESS SCALAR FIELD WITH TYPE B SELF-SIMILARITY}

For the Type B self-similar solutions, we find that the non-vanishing components of the Ricci tensor are given by

$$
\begin{aligned}
& R_{u u}=\frac{z}{u^{2}}\left[z\left(U^{\prime \prime}-\frac{1}{2} U^{\prime 2}\right)+\left(2+z M^{\prime}\right) U^{\prime}\right] \\
& R_{u v}=\frac{1}{u^{2}}\left[z\left(M^{\prime \prime}+U^{\prime \prime}-\frac{1}{2} U^{\prime 2}\right)+M^{\prime}+U^{\prime}\right] \\
& R_{v v}=\frac{1}{u^{2}}\left(U^{\prime \prime}-\frac{1}{2} U^{\prime 2}+U^{\prime} M^{\prime}\right) \\
& R_{x x}=R_{y y}=-\frac{z}{u^{2}} e^{M-U}\left(U^{\prime \prime}-U^{\prime 2}+\frac{U^{\prime}}{z}\right)
\end{aligned}
$$

One can show that the corresponding scalar field has to take the same form, Eq.(3.6). Since $\phi=\phi(u, v)$, we find that $R_{x x}=\phi_{, x}^{2}=0$, which together with Eq.(4.4) yields

$$
U^{\prime \prime}-U^{\prime 2}+\frac{U^{\prime}}{z}=0
$$

This equation has the general solution

$$
U=-\ln [\alpha \ln (z)+\beta],
$$

where $\alpha$ and $\beta$ are two integration constants. On the other hand, it can be shown that now Eq.(2.6) becomes, 


$$
\left(z \varphi^{\prime}\right)^{\prime}+\left(q-z \varphi^{\prime}\right) U^{\prime}=0
$$

The general solution of it is given by

$$
\varphi(z)=q \ln (z)+\gamma \ln [\alpha \ln (z)+\beta]+\varphi_{0},
$$

where $\gamma$ and $\varphi_{0}$ are arbitrary constants. Inserting Eqs.(3.6), (4.3), (4.6) and (4.6) into the Einstein field equations for the $v v$-component, we find that

$$
M^{\prime}=\frac{1-2 q \gamma}{z}+\frac{\alpha\left(1-2 \gamma^{2}\right)}{2 z[\alpha \ln (z)+\beta]}-\frac{q^{2}}{\alpha z}[\alpha \ln (z)+\beta],
$$

which has the solution

$$
M(z)=(1-2 q \gamma) \ln (z)+\frac{1}{2}\left(1-2 \gamma^{2}\right) \ln \{[\alpha \ln (z)+\beta]\}-\frac{q^{2}}{2 \alpha^{2}}[\alpha \ln (z)+\beta]^{2}+M_{0},
$$

where $M_{0}$ is another integration constant. Substituting the solutions into the rest of the Einstein field equations, we find that the constant $\gamma$ must be given by,

$$
\gamma=\frac{1}{2 q}
$$

Then, the general solutions are given by

$$
\begin{aligned}
U(z) & =-\ln [\alpha \ln (z)+\beta], \\
M(z) & =-\frac{1}{4 q^{2}}\left(1-2 q^{2}\right) \ln \{[\alpha \ln (z)+\beta]\}-\frac{q^{2}}{2 \alpha^{2}}[\alpha \ln (z)+\beta]^{2}+M_{0}, \\
\varphi(z) & =q \ln (z)+\frac{1}{2 q} \ln [\alpha \ln (z)+\beta]+\varphi_{0} .
\end{aligned}
$$

From the above expressions we can see that to have the solutions valid near the hypersurface $v=0$ in the region $u \leq 0, v \geq 0$, we must assume

$$
\alpha<0 .
$$

To study the spacetime near the null hypersurface $v=0$ further, following the discussions given in Sec.III.B, one can show that now the hypersurface $v=0$ also represents a past null infinity. This null infinity is free of spacetime singularity. This can be seen, for example, from the Ricci scalar, which now is given by

$$
\begin{aligned}
R= & \phi_{, \alpha} \phi^{, \alpha}=-\frac{4 q^{4}[\alpha \ln (z)+\beta]^{2}-\alpha^{2}}{2 q^{2} z u^{2}[\alpha \ln (z)+\beta]^{\left(1+6 q^{2}\right) / 4 q^{2}}} \\
& \times \exp \left\{M_{0}-\frac{q^{2}}{2 \alpha^{2}}[\alpha \ln (z)+\beta]^{2}\right\} .
\end{aligned}
$$

Thus, $v=0$ is actually a past null boundary of the spacetime. On the other hand, from Eq.(4.14) we can see that the spacetime is singular on the hypersurface

$$
z=z_{0} \equiv e^{-\beta / \alpha}
$$

which is spacelike and serves as the up boundary of the spacetime. The corresponding Penrose diagram is given by Fig. 2. However, unlike the case discussed in Sec.III.B, now the scalar field is spacelike near the null hypersurface $v=0$. Therefore, the corresponding solution cannot be interpreted as representing gravitational collapse of the scalar field in the region $u<0, v>0$. 


\section{CONCLUSIONS}

In this paper, we have studied plane symmetric self-similar solutions to Einstein's four-dimensional theory of gravity and found all such solutions. We have also studied the local and global properties of those solutions and found that some of them can be interpreted as representing gravitational collapse of the scalar field. During the collapse, trapped surfaces are never developed. As a result, no black hole is formed. Although the collapse always ends with spacetime singularities, it is found that these singularities are spacelike and not naked.

An interesting question is: Do these solutions represent critical collapse? To answer this question, one needs to study the linear perturbations of the solutions and show that there exists one solution that has one and only one unstable mode. Such a study is out of the scope of this paper, and we wish to return to this problem in another occasion.

[1] B. J. Carr and A. A. Coley, Class. Quant. Grav. 16, R31 (1999); H. Maeda, T. Harada, H. Iguchi, and N. Okuyama, Prog. Theor. Phys. 108, 819 (2002); 110, 25 (2003); B. J. Carr and C. Gundlach, Phys.Rev. D67, 024035 (2003).

[2] M. W. Choptuik, Phys. Rev. Lett. 70, 9 (1993); "Critical Behavior in Massless Scalar Field Collapse," in Approaches to Numerical Relativity, Proceedings of the International Workshop on Numerical Relativity, Southampton, December, 1991, Edited by Ray d'Inverno (Cambridge University Press, Cambridge, 1992); "Critical Behavior in Scalar Field Collapse," in Deterministic Chaos in General Relativity, Edited by D. Hobill et al. (Plenum Press, New York, 1994), pp. $155-175$.

[3] C. Gundlach, "Critical phenomena in gravitational collapse: Living Reviews," gr-qc/0001046 (2000), and references therein.

[4] A.Z. Wang, "Critical Phenomena in Gravitational Collapse: The Studies So Far," gr-qc/0104073, Braz. J. Phys. 31, 188 (2001).

[5] A.Z. Wang, Phys. Rev. D68, 064006 (2003).

[6] D. Garfinkle, Phys. Rev. D63, 044007 (2001).

[7] F. Pretorius and M. W. Choptuik, Phys. Rev. D62, 124012 (2000).

[8] V. Husain and M. Olivier, Class. Quantum Grav. 18, L1 (2001).

[9] D. Garfinkle and C Gundlach, Phys. Rev. D66, 044015 (2002).

[10] E.W. Hirschmann, A.Z. Wang, and Y. Wu, "Collapse of a Scalar Field in 2+1 Gravity," gr-qc/0207121 (2002).

[11] C. R. Evans and J. S. Coleman, Phys. Rev. Lett. 72, 1782 (1994); T. Koike, T. Hara, and S. Adachi, ibid., 74, 5170 (1995); C. Gundlach, ibid., 75, 3214 (1995); E. W. Hirschmann and D. M. Eardley, Phys. Rev. D52, 5850 (1995).

[12] D. Kramer, H. Stephani, E. Herlt, and M. MacCallum, Exact Solutions of Einstein's Field Equations (Cambridge University Press, Cambridge, England, 1980).

[13] D. Tsoubelis and A.Z. Wang, Gen. Relativ. Grav., 22, 1091 (1990).

[14] M.E. Cahill and A.H. Taub, Commun. Math. Phys. 21, 1 (1971).

[15] A.H. Taub, "Plane-Symmetric Similarity Solutions for Self-Gravitating Fluid," in General Relativity, papers in honour of J.L. Synge, edited by L. O'Raifeartaigh (Clarendon Press, Oxford, 1972), pp. 133-150. 